\title{
Law enforcement agencies' approach to de-escalation: Incorporating a social services perspective
}

\author{
Lisa M. Deveau*
}

This article is related directly to the $6^{\text {th }}$ International Law Enforcement $\mathcal{E}$ Public Health (LEPH) Virtual Conference in March 2021

\begin{abstract}
In this critical review and social innovation narrative, we analyze the literature regarding Canadian law enforcement agencies' approach to de-escalation and crisis intervention. Using an interdisciplinary approach, we consider how the skills and values of social work can be used to inform and train officers on essential skills such as de-escalation and conflict resolution. We look at the systemic barriers to bringing about change within Canadian police forces as the current culture continues to be influenced by colonization and law enforcement continues to value and endorse use of force over de-escalation. While services can benefit by applying an interdisciplinary lens when training officers, the factors that impede this union and collaboration are discussed and explored as police services are given immense discretion in how they train and respond to mental health crises. In conclusion, we examine the government's role in perpetuating these issues.
\end{abstract}

\section{INTRODUCTION}

Crisis intervention, defined as supporting individuals and families in times of extreme duress, is an essential service provided by many professional bodies. Police officers are among those who respond. Mental health calls make up a large percentage of the calls for service to which law enforcement officers respond. In these circumstances, first responders are the first point of contact in de-escalating members of the public in crisis and restoring order. This literature review aims to contribute to guidelines for officers' response to mental health by identifying the best and most promising practices used in other disciplines that may benefit police officers in de-escalating mental health calls. The literature review has been conducted with the following questions in mind: What are the theories that inform both social services and law enforcement practices? What are the skills used by social workers and affiliated mental health professionals in de-escalating mental health crises? Can these skills be used by law enforcement officers and their approach in de-escalation? How might citizens' perception of officers hinder the application of these skills? Finally, we will discuss the research questions that have yet to be answered.
Social Services Theoretical Framework and Skills

The reviewed literature is rooted in social services and humanities disciplines and includes scholarly articles from psychiatric nursing, social work, and psychology. The theories within these disciplines create frameworks from which skills are derived and subsequently applied. A common theory discussed in the literature is symbolic interaction.

The theory of symbolic interaction states that interactions are dependent on both parties' perceptions of the world around them and explores how prior beliefs inform subsequent meanings (Berring et al., 2016). According to Berring et al. (2016), symbolic interactionism has three main premises: 1) Humans act towards events depending on the meaning they have for them; 2) People have different meanings; and 3) Meanings can change. In any interaction, there is the person who relays the information and the person who decodes the message. Research has shown that conflict and aggression emerge when there is distrust, which generally occurs when a person's interpretation of an action is incompatible with their expectation (Berring et al., 2016; Richter et al., 2006, p. 127). For example, a person who views police as helpful but then finds their actions intimidating and coercive will begin

Correspondence to: Lisa Deveau, Department of Social Work, Carleton University, 1125 Colonel By Drive, Ottawa, ON K16 5B6, Canada. E-mail: lisa.deveau@outlook.com. To cite: Deveau, L. (2021). Law enforcement agencies' approach to de-escalation: Incorporating a social services perspective. Journal of Community Safety and Well-Being, 6(2), 66-70. https://doi.org. 10.35502/jicswb.182

@ Author(s) 2021. Open Access. This work is distributed under the Creative Commons BY-NC-ND license. For commercial re-use, please contact sales@sgpublishing.ca. gg PUBLISHING Published by SG Publishing Inc. CSKA Official publication of the Community Safety Knowledge Alliance. 
to distrust the police and simultaneously believe they are in danger. According to Richter et al. (2006), apart from patients with specific personality disorders, human beings are usually not voluntarily aggressive; aggression and violence are triggered by the subjective notion that one must defend oneself against intimidating or unjust behaviours. Breaking this "paranoid" thinking is the key task in non-violent conflict resolution (Richter et al., 2006).

Drawing from the literature, the skills most commonly practised and encouraged by social workers and allied professionals are self-awareness, self-reflection, and emotional regulation. Within social services, the emphasis is placed on the practitioner to analyze, recognize, and respond to the situation.

\section{Skills in Social Services}

Self-awareness is a powerful tool in achieving de-escalation, as it encourages the practitioner to consider how they present themselves and whether they are displaying coercive tactics or an abuse of power; both coercion and power have been linked to aggressive outbursts from patients (Berring et al., 2016). Patients who feel powerless develop feelings of fear and resentment due to a lack of respect (Berring et al., 2016, p. 500; Richter et al., 2006, p. 127; Nordstrom et al., 2012, p. 4). Self-awareness encourages the practitioner to consider the verbal and non-verbal cues being displayed and how the patient might perceive these actions, while simultaneously considering the patient's past experiences that might also influence their interpretation. Practitioners who were most effective in de-escalation created a safe space by showing mutual respect, displaying empathetic gestures, and relinquishing power (Berring et al., 2016, p. 505; Duperouzel, 2008, p. 303; Richter et al., 2006, p. 125; Nordstrom et al., 2012, p. 5).

Self-reflection is another skill and is helpful in achieving self-awareness as it allows the practitioner to consider their behaviours and beliefs following the interaction, and the ways in which they contributed to the patient's response (Berring et al. 2016, p. 505; Duperouzel, 2008, p. 303; Richter et al., 2006, p. 128). For example, (psychiatric) patients are concerned with short-term goals, and when this "need" is threatened and their sense of security is in jeopardy, it acts as a trigger for violent outbursts (Richter et al., 2006, p. 127). In reflecting on why a homeless individual appears overly protective of their possessions and thus uncooperative, one may consider that the individual has lost their belongings and the items are the last of their possessions. Self-reflection encourages the practitioner to consider how they presented themselves and the daily struggles of the person.

Another skill commonly used in social work is emotional regulation. Practitioners remain aware of their emotional responses towards individuals, and control and adjust their reactions. Practitioners are capable of acknowledging that some actions are outside of the person's control, and, in doing so they are less likely to become defensive, judgemental, and antagonizing (Duperouzel, 2008, p. 306; Richter et al., 2006, p. 128; Nordstrom, 2006, p. 6). According to Duperouzel (2008), practitioners will put on a "facade," appearing calm among chaos. Emotional regulation is about maintaining composure even when challenged.

Mental health professionals approach de-escalation by emphasizing and mastering these skills. Law enforcements' response to mental health would benefit by applying an interdisciplinary research approach. It is important to consider the theoretical frameworks that influence law and security when attempting to merge social services with enforcement.

\section{Policing Theoretical Framework}

In contrast to social services approaches, policing, law, and security apply de-escalation from an enforcement model. Policing differs from social services and humanities in that officers are permitted to use "lawful force" to regain control. In several ways, warranted force is the essence of policing and is used as a tactic to restore public order (Fielding, 2002). The issue with "use of force," is that it is subjective in nature, as the application of force is discretionary and often contingent on officer perception and tolerance (Fielding, 2002). Force can be prematurely used depending on an officer's temperament, thus creating issues around excessive force and what constitutes legitimate force (Fielding, 2002).

Furthermore, the enforcement model tells officers that citizens must respect police by their mere presence, power, and authority. When their power is challenged, which is especially likely in mental health crises, it creates an issue for the responding officer, who has been conditioned to believe that their verbal commands should not be challenged. This conflicting narrative creates a struggle for officers when they fail to gain the compliance that was expected. In order to regain control, officers use the skills they have been taught, which no doubt stem from an enforcement and "use of force" model and involve forceful tactics, including electronic control devices (taser), batons, oleoresin capsicum (OC) spray, combative strikes, and firearms.

Expectations theory is also worth discussing in relation to policing as there is a hierarchy within law enforcement agencies. Expectations theory states that within organizations, members assume or are appointed certain roles, resulting in a hierarchy among staff. For various reasons, specific individuals are implicitly deemed as more valuable and are offered more responsible roles while other employees adhere to submissive directives (Correll \& Ridgeway, 2003). According to Correll \& Ridgeway (2003), one of the ways in which members are "selected" to carry out specific tasks is through "status characteristics." Status characteristics are social constructs presumed to anticipate the quality of a member's future work. For example, a status characteristic can be the assumption that men are better at technological tasks than women; men, therefore, would automatically assume supervisory roles within technological companies while women assume junior positions (Correll \& Ridgeway, 2003). In relation to law enforcement, the emphasis on using force to regain control and compliance has resulted in law enforcement placing more value on men as they are seen as "masculine" and "intimidating"; this has meant, historically, that they were hired as officers, and, in the present time, that they have assumed more dominant/senior positions in the force.

Both theories are important to consider when looking at the culture of policing. Law enforcement values "legitimate force" over de-escalation skills, and those who hold supervisory positions further endorse and value "use of force." Forces are quick to invest money into weaponized supplies and slow to invest in crisis and negotiation training. While some services incorporate Crisis Intervention Training (CIT) 
that educates and encourages officers to use de-escalation and communication skills, this approach needs to be encouraged and practised unanimously across Canadian police forces. Currently, each service has discretion in the number of hours they dedicate to crisis intervention and de-escalation training.

\section{De-escalation Skills Taught in Policing}

The research record is inadequate in identifying promising skills taught and used by police officers to de-escalate mental health calls. The research is outdated and insufficient to draw any conclusions, mostly evaluating the success of deescalation by focusing on reduced arrest rates, community referrals, and reduced on-scene time. Officers certainly benefit by either working with mental health professionals or being trained from a social services perspective, such as CIT, as arrest rates are lowered, times on scene responding are reduced, and community resources are more likely to be referred to persons in crisis (Oliva et al., 2010; Watson et al., 2008; Coleman \& Cotton 2010; Wortley et al., 2006). However, these outcomes do not equate to effective de-escalation skills in which force was not used by an officer, and more research is needed in this area to draw conclusions.

\section{Tying Them Together}

Law enforcement has the potential to improve responses to mental health by incorporating social service research and skills when training officers. It is important that officers be equipped with essential skills to de-escalate mental health crises. Patients in crisis prefer when mental health professionals accompany police, reporting that they have a more positive interaction where coercive and forceful tactics are less likely to be used (Kisely et al., 2010; Lamanna et al., 2015; Kirst et al., 2015; Canada et al., 2019; Nicholson \& Marcoux, 2018). Furthermore, it has been concluded that almost half of all police-involved fatalities occurring in Canada, since 2000, involved a person who was under mental duress; another $48 \%$ of the interactions involved comorbid circumstances where the person was mentally distressed and/or under the influence of a substance (Nicholson \& Marcoux, 2018).

When incorporating non-combative skills, how does one effectively incorporate social work strategies into the educational policies that govern law enforcement? How will officers accept this multidisciplinary approach in responding to mental illness? Research has found education to be an effective tool in changing behaviours and beliefs. Educational seminars, discussions, and scenario-based training that incorporate social services skills and encourage officers to self-reflect, be self-aware, and practise emotional regulation will undoubtedly help them de-escalate and diffuse a person in crisis. Interdisciplinary research can also help explain the behaviours of a person in crisis. For example, shouting often occurs when a person feels their opinion is not heard and is not necessarily indicative of challenging someone. Therefore it is important to acknowledge what a person is saying. Social work also applies an intersectional lens in relation to mental distress, incorporating and considering the social determinants of health and how this impacts a citizen's well-being and mental health.

It is not enough to simply educate officers who work on the frontline about mental health, de-escalation, and conflict resolution. As previously discussed, the culture and hierarchy within policing need to be considered. The current culture of policing preserves the status quo by recruiting, training, and socializing officers in a very traditional manner, orienting them towards an enforcement model. Research has shown that, in order to effectively implement change, upper management, administrators, sergeants, and staff sergeants need to adopt an optimistic attitude towards change (Novak et al., 2003). As discussed by Novak et al. (2003), those who create the policy are not responsible for implementing the policy, as frontline officers have the opportunity to choose how they will react to organizational policies and decide whether to comply, ignore, or even sabotage them (Novak et al., 2003, p. 7). Unity among upper management is of paramount importance in adopting and embracing new strategies that are then introduced to fellow officers (Novak et al., 2003, p. 7). Subordinate officers look to their sergeants, staff sergeants, inspectors, and the chief for praise and honorariums. Thus their support, in a non-militant style of policing, is needed for "junior" (lower-ranking) officers to adopt and use these skills (Novak et al., 2003, p. 7).

\section{Other Factors and De-Escalation}

When understanding policing and de-escalation, there are other variables that influence the interaction and the outcome, such as gender, race, and educational background. Research has shown that women police officers are less likely to use force and tend rather to rely on communication and deescalation techniques; they are subject to fewer allegations of excessive force (Rabe-Hemp \& Schuck, 2007; Schuck \& RabeHemp, 2007). Yet, research has shown no difference in assaultive behaviours towards female officers compared with male officers, except when responding to calls involving domestic violence, where female officers are assaulted more than their male coworkers (Rabe-Hemp \& Schuck, 2007; Schuck \& RabeHemp, 2007). Despite this, female officers consciously avoid using force even when it may be warranted. According to Schuck \& Rabe-Hemp (2007), female officers are more likely to engage in "unpredictable policing," that is, using communication and de-escalation, in situations where one might have predicted the use of force. Conversely, male officers were more likely to practise "overpredicted policing," in which force was used pre-emptively (Schuck \& Rabe-Hemp, 2007). Force is not always necessary in gaining compliance-women often effectively achieve this while relying on de-escalation and conflict resolution.

Another factor discussed within the research was race and whether it plays a role in how citizens perceive police. In one study, officer race, ethnicity, age, and physical size were found to be insignificant in how citizens perceive officers and did not influence the frequency with which force was used against the officer or by an officer (Weitzer, 2000). Most research has shown that it is the demographics of the neighborhood rather than the individual's race that has an impact on how officers are perceived (Weitzer, 2000). Low socioeconomic neighborhoods are more likely to have a negative perception of police, seeing them as more coercive and aggressive, regardless of race, and higher socioeconomic neighborhoods view police more favourably (Weitzer, 2000). The research focused on perception and not necessarily force used.

Supportive actions, including de-escalation and communication, are significantly influenced by an officer's educational 
background. Officers with post-secondary education are more likely to engage in supportive actions, such as communication, than those without post-secondary education (Weitzer, 2000). Furthermore, officers who have shown a vested interest in mental health, either through educational endeavours, voluntary actions, or knowing someone with mental health concerns, are more likely to engage in de-escalation and conflict resolution.

\section{What is Missing in the Research?}

The research fails to consider the reasons why "use of force" continues to be accepted and prioritized by law enforcement agencies. Currently, law enforcement dedicates most of its time training recruits/officers on efficient use of their weapons, such as firearms, electronic control devices (taser), batons, and oleoresin capsicum (OC) spray. Officers receive full-day, in-class, hands-on training that is dedicated to effective tactical techniques. Yet, the same level of training is not mandated nor encouraged for non-combative, conflict resolution, and de-escalation training. The current research does not adequately explore why agencies continue to practise from an enforcement model rather than a community approach, especially given the shift in officers' duties as they continue to take on a more direct role in mental health calls. Throughout an officer's career, they are far more likely to be required to use communication and de-escalation than force, yet law enforcement continues to train using an enforcement model.

Further, while consolidating the research, it was unclear whether researchers examined and considered how the designation of a mental health professional influenced the citizen's perception and thus the interaction. Were citizens more compliant when mental health professionals accompanied police due to the fact that they knew they were speaking to an "expert" versus an officer? Since mental health professionals self-identify, did this act almost as a placebo effect? Was it actually the professional skills of the mental health professional or was it a combination of those skills and their professional designation? This was not adequately discussed in the research and can shed light on how citizens' perceptions influence the outcome of a mental health crisis. If officers hold multiple designations (for example, if they are a registered social worker while also being a police constable), should this be revealed to citizens as it may facilitate the interaction during mental health calls?

The research does not explore the various factors that influence de-escalation, such as gender, race, and education, and how law enforcement agencies plan to mitigate and address these issues. If female and educated officers are more likely to engage in de-escalation and conflict resolution, are educated and female officers more likely to be recruited into units that specifically respond to mental health crises? What are the skills and responses that make these officers more effective in de-escalating mental health crises?

\section{CONCLUSION}

Having discussed de-escalation and how it is taught and applied within law enforcement, specifically in relation to mental health calls, we have shown that there needs to be an amendment. Law enforcement agencies would benefit from applying an interdisciplinary approach to training officers. By incorporating the skills used by mental health professionals, officers may learn self-awareness, self-reflection, and emotional regulation, skills that are promising in de-escalating mental health crises.

\section{ACKNOWLEDGEMENTS}

I would like to acknowledge Dr. Susan Braedley, my supervisor for the Crisis Intervention and Policing course I created.

\section{CONFLICT OF INTEREST DISCLOSURES}

The author has no conflicts of interest to declare.

\section{AUTHOR AFFILIATIONS}

*Department of Social Work, Carleton University, Ottawa, ON, Canada.

\section{REFERENCES}

Berring, L., Pedersen, L., \& Buus, N. (2016). Coping with violence in mental health care settings: Patient and staff member perspectives on de-escalation practices. Archives of Psychiatric Nursing, 30151, 499-507. https://doi.org/10.1016/i.apnu.2016.05.005

Canada, K., Barrenger, S., Ray, B., \& Canada, K. (2019). Bridging mental health and criminal justice systems: A systematic review of the impact of mental health courts on individuals and communities. Psychology, Public Policy, and Law, 25(2), 73-91. https://doi.org/10.1037/ law0000194

Coleman, T., \& Cotton, D. (2010). Reducing risk and improving outcomes of police interactions with people with mental illness. Journal of Police Crisis Negotiations: The Police and People with Mental Illness: New Approaches to a Longstanding Problem, 10(1-2), 39-57. https:// doi.org/10.1080/15332581003756950

Correll, S. J., \& Ridgeway, C. L. (2006). Expectation states theory. In Handbook of social psychology (pp. 29-51). Springer, Boston, MA.

Duperouzel, H. (2008). "It's OK for people to feel angry": The exemplary management of imminent aggression. Journal of Intellectual Disabilities, 12(4), 295-307. https://doi.org/10.1177/1744629508100495

Fielding, N. G. (2002). Theorizing community policing. British Journal of Criminology, 42(1), 147-163.

Nordstrom, K., Zun, L. S., Wilson, M. P., Stiebel, V., Ng, A. T., \& Bregman, B. (2012). Medical evaluation and triage of the agitated patient: Consensus statement of the American Association for Emergency Psychiatry Project BETA Medical Evaluation Workgroup. Western Journal of Emergency Medicine, 13(1), 3-10. https://doi.org/10.5811/ westjem.2011.9.6863

Lamanna, D., Kirst, M., Shapiro, G., Matheson, F., Nakhost, A., \& Stergiopoulos, V. (2015). Toronto Mobile Crisis Intervention Team (MCIT): Outcome evaluation report. St. Michaels Hospital.

Novak, K., Alarid, L., \& Lucas, W. (2003). Exploring officers' acceptance of community policing: Implications for policy implementation. Journal of Criminal Justice, 31(1), 57-71. https://doi.org/10.1016/500472352/02/00199-X

Nicholson, K., \& Marcoux, J. (2000). Most Canadians killed in police encounters since 2000 had mental health or substance abuse issues. CBC News. https://www.cbc.ca/news/investigates/mostcanadians-killed-in-police-encounters-since-2000-had-mental-healthor-substance-abuse-issues-1.4602916

Oliva, J., Morgan, R., \& Compton, M. (2010). A practical overview of deescalation skills in law enforcement: Helping individuals in crisis while reducing police liability and injury. Journal of Police Crisis Negotiations, 10(1-2), 15-29. https://doi.org/10.1080/15332581003785421

Price, O., Baker, J., Bee, P., \& Lovell, K. (2015). Learning and performance outcomes of mental health staff training in de-escalation techniques 
for the management of violence and aggression. The British Journal of Psychiatry, 206(6), 447-455. https://doi.org/10.1192/bip. bp. 114.144576

Richter, D., \& Whittington, R. (2006). Nonphysical conflict management and de-escalation. In Richter, D. (Eds.). Violence in mental health settings: Causes, consequences, management(pp. 125-144). Springer Science \& Business Media, LLC.

Rabe-Hemp, C. E., \& Schuck, A. M. (2007). Violence against police officers: Are female officers at greater risk? Police Quarterly, 10(4), 411-428. https://doi.org/10.1177/1098611107304326

Schuck, A., \& Rabe-Hemp, C. (2007). Women police: The use of force by and against female officers. Women \& Criminal Justice, 16(4), 91-117. https://doi.org/10.1300/J012v16n04_05
Watson, A., Morabito, M., Draine, J., \& Ottati, V. (2008). Improving police response to persons with mental illness: A multi-level conceptualization of CIT. International Journal of Law and Psychiatry, 31 (4), 359-368. https://doi.org/10.1016/i.iilp.2008.06.004

Weitzer, R. (2000). White, black, or blue cops? Race and citizen assessments of police officers. Journal of Criminal Justice, 28(4), 313-324. https://www.sciencedirect.com/science/article/abs/pii/ S004723520000043X? via\%3Dihub

Wortley, S. (2006). Police use of force: As examination of data from the special investigations unit. University of Toronto. https://www. attorneygeneral.jus.gov.on.ca/inquiries/ipperwash/policy_part/ projects/pdf/AfricanCanadianCliniclpperwashProject_SIUStudyby ScotWortley.pdf 